\title{
Conventional vs Robotic Arm Assisted Total Hip Arthroplasty (THA) Surgical Time, Transfusion rates, Length of Stay, Complications and Learning Curve
}

\author{
Yi Ying Heng ${ }^{1^{*}}$, Rajitha Gunaratne ${ }^{1,2}$, Charlie Ironside ${ }^{2}$ and Arash Taheri ${ }^{1,3}$ \\ ${ }^{1}$ Joondalup Health Campus, Shenton Avenue, Joondalup, WA, 6027, Australia \\ ${ }^{2}$ Curtin University, Kent Street, Bentley, WA, 6102, Australia \\ ${ }^{3} J o o n d a l u p$ orthopaedic group, 60 Shenton Avenue, Joondalup, WA, 6027, Australia
}

"Corresponding author: Heng Yi Ying, Joondalup Health Campus, Shenton Avenue, Joondalup, WA, 6027, Australia, Tel: +61411793009; E-mail: hengyiying@gmail.com

Received date: July 29, 2018; Accepted date: August 05, 2018; Published date: August 07, 2018

Copyright: () 2018 Heng YY, et al. This is an open-access article distributed under the terms of the Creative Commons Attribution License, which permits unrestricted use, distribution, and reproduction in any medium, provided the original author and source are credited.

\begin{abstract}
Background: Total hip arthroplasty (THA) is increasingly more popular with our aging population. Robotic arm assisted THA uses patient specific information gathered from a pre-operative CT scan and correlation with intraoperative checkpoints to improve the accuracy and reproducibility of component positioning. Despite the various advantages robotic arm assisted THA can offer, there are still concerns regarding increased surgical time, technical complexity, complications and costs.
\end{abstract}

Method: Retrospective review of a single surgeons' last 45 conventional THA performed prior to changing to the robotic arm assisted system with the first 45 robotic arm assisted THA. Surgical time, Length of stay (LOS) in hospital, LOS in rehabilitation, transfusion rates and any complications were compared.

Results: Average surgical time was 96.7 mins for the robotic group and 84.9 mins for conventional group; however each robotic operation was approximately one minute shorter than the previous operation and the average time for the last 10 cases reduced to 82.9 mins. Compared to conventional THA there is no increased risk of complications or transfusions and interestingly there may be less chance of intraoperative acetabular fractures due to the single ream, minimal bone resection technique. LOS in the robotic group that did not go to rehabilitation was shorter by approximately 1 day and although statistically analyse for LOS in rehabilitation was not done due to small numbers there was a tendency for shorter LOS in the robotic group as well.

Conclusion: This reduction in LOS, comparable surgical times and potential for less complications may outweigh the increased initial costs associated with the robotic system.

Keywords: Robotic THA; MAKO; Robotic arm assisted arthroplasty

\section{Introduction}

Total hip arthroplasty (THA) is increasingly needed with our aging population [1] as it is the only successful interventions in treating the morbidity of patients with end-stage hip osteoarthritis [2]. The incidence of primary THR in USA is predicted to increase by $174 \%$ by 2030 [2]. Despite advances in technology and implants, technical or surgical errors potentially account for up to $40 \%$ of revision hip arthroplasty [3]. Malposition of acetabular and femoral components and inadequate restoration of hip offset and center of rotation may result in accelerated prosthetic wear, instability, early implant failure and poor functional outcomes [4-9].

Traditionally, surgeons utilize bone and soft tissue landmarks, alignment guides and their own clinical expertise for component positioning [9]. Robotic arm assisted THA uses patient specific information gathered from a pre-operative CT scan and correlation with intraoperative checkpoints to improve the accuracy and reproducibility of component positioning [10-12]. During surgery, the femur and acetabulum are correlated with the CT using three landmark points and 32 registration points for each. The accepted registration error is less than or equal to $0.5 \mathrm{~mm}$.

Studies have shown that robotic arm assisted THA have more precise component positioning than patients who had a standard THA [13-15]. This is particularly useful in patients with altered anatomy [10]. Despite the various advantages robotic arm assisted THA can offer, there are still concerns regarding increased surgical time, technical complexity, complications and costs [16-18].

The purpose of this study was to analyze 1) operative time 2) blood transfusion rates 3 ) length of stays in hospital 4) complication rates and 5) learning curve to quantify clinically relevant differences in outcomes following standard total hip arthroplasty compared with robotic arm assisted surgery.

\section{Method}

This study was approved by our institutional ethics review board. We retrospectively reviewed the clinical experience of a single surgeon at one institution. The surgeons last 45 conventional THA performed 
prior to changing to the robotic arm assisted system were compared with the first 45 robotic arm assisted THA. The robotic system used was the fully enhanced MAKO robotic hip system (MAKO Stryker, Kalamazoo, Michigan). Only primary uncemented THA for osteoarthritis were included and the same posterior approach and implant was used in both groups. The robotic system intraoperatively correlates the femur and acetabulum with a preoperative CT. This is used to navigate the neck cut, measure the broach anteversion and use the robotic arm to ream the acetabulum with a single ream plus position the cup to achieve a desired combined anteversion.

All included patients' files were reviewed by two authors. Surgical time was obtained from the patients' surgical record sheets, which showed the time surgery started and ended. Surgical start time was standardised as time the limb was being prepared, while end time was when dressings were put on. Length of stay (LOS), transfusion rates and any perioperative or postoperative complications such as dislocations, periprosthetic fractures, readmissions, deep infections and thromboembolic events, were extracted by reviewing patient files. The operation reports, anaesthetic charts, outpatient notes and imaging up to 3 months post-surgery were reviewed by the authors. We looked at the length of in-patient hospital stay between the two groups, and within each group, we studied the patients who required in-patient rehabilitation in a rehabilitative unit and patients who did not require further rehabilitation.

Categorical data were analyzed using chi-square tests to determine whether there were any differences between groups. Independent samples t-tests were conducted to analyze continuous variable data, with effect sizes for t-tests estimated using Cohen's d (small $=0.20$, medium $=0.50$, large $=0.80$ ). All continuous data was assessed for outliers (more than three standard deviations above or below the mean) and when these are found a note is made but no data has been excluded.

\section{Results}

Descriptive statistics are presented in Table 1.

\section{Baseline differences}

There was no difference between groups in age at the time of surgery, hip side, or gender. The groups are thus considered equivalent at baseline.

\section{Surgical time (minutes)}

The average surgical time for the robotic group was 96.7 mins, whereas the average surgical time for the manual group was 84.9 mins.

\section{Transfusion rates}

There were not enough patients who had a transfusion for this to be analyzed statistically as only one patient in each group needed a transfusion. There does not appear to be any significant difference in both groups otherwise.

\section{Length of stay}

There was no effect of group on overall length of stay, $t(88)=1.28$, $\mathrm{p}=0.21$. There were not enough patients who went to a rehabilitation ward to statistically analyse LOS in rehabilitation. There were five patients who went to rehabilitation in the conventional group and four patients in the robotic group. The average total LOS for these patients who went to rehabilitation was 22.4 days for the conventional group and 17.0 days for the robotic group. Once we excluded patients who required rehabilitation, then there are no longer any outliers present in either the conventional group or the robotic group. There was an effect of group on LOS, where the Conventional group had longer LOS compared to the Robotic group, $\mathrm{t}(79)=2.73, \mathrm{p}=0.008, \mathrm{~d}=0.61$.

\section{Complications}

There were 3 intraoperative complications in the conventional group; all 3 were minor acetabular fractures while implanting the cup which required screw fixation of the cup for extra stability. The robotic group had 1 intraoperative complication of software malfunction requiring conversion to conventional methods. There was 1 postoperative superficial wound infection requiring return to theatre for wound washout and debridement in the conventional group and no postoperative complications in the robotic group. Complications were not analyzed statistically as there were low numbers.

\begin{tabular}{|l|l|l|}
\hline & Conventional & Robotic \\
\hline $\begin{array}{l}\text { Age } \\
\text { (years) }\end{array}$ & $62.8(12.3)$ & $64.5(9.9)$ \\
\hline Side & Left=21, Right=24 & Left=20, Right=25 \\
\hline Gender & $\mathrm{M}=32, \mathrm{~F}=13$ & $\mathrm{M}=25, \mathrm{~F}=20$ \\
\hline Surgical time (minutes) & $84.9(30.7)$ & $96.7(20.1)$ \\
\hline Transfusion? & Yes=1, No=44 & Yes=1, No=44 \\
\hline Length of stay: Overall & $5.93(6.95)$ & $4.22(5.70)$ \\
\hline Length of stay: Non-rehabilitation & $3.88(1.40)$ & $2.98(1.56)$ \\
\hline
\end{tabular}

Table 1: Descriptive statistics for continuous variables include mean with standard deviation in brackets while counts are included for categorical variables.

\section{Learning effect on surgical time (minutes)}

To determine whether there was an improvement in surgical time over subsequent operations, a linear function was fit to the ordered operation data (see Figure 1).

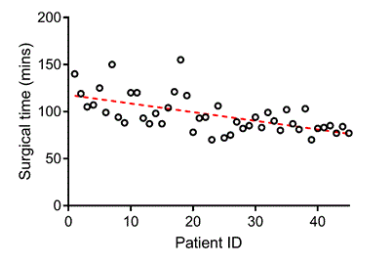

Figure 1: Surgical time in minutes as a function of the order operations were performed in is presented in hollow circles. A linear function fit is represented by the red dashed line $\left(\mathrm{Y}=-0.92^{\star} \mathrm{X}\right.$ $+117.8)$.

The slope of the line was significantly different from zero, $\mathrm{F}(1,43)=24.1, \mathrm{p}<0.001$, suggesting that there was a reduction in duration over subsequent operations. Each operation was 
Citation: Heng YY, Gunaratne R, Ironside C, Taheri A (2018) Conventional vs Robotic Arm Assisted Total Hip Arthroplasty (THA) Surgical Time, Transfusion rates, Length of Stay, Complications and Learning Curve. J Arthritis 7: 272. doi:10.4172/2167-7921.1000272

Page 3 of 4

approximately one minute shorter $(-0.92 \mathrm{mins})$ than the previous operation.

\section{Discussion}

Robotic arm assisted THA is an exciting new technology [19], which has proven to be a useful tool for accurate and reproducible component positioning [9-13]. Recently published studies by Bukowski et al. [9] and Perets et al. [20] have also reported improvements in patient reported outcomes such as pain, function and overall wellbeing.

Integration of the technology into surgical practice is still slow due to concerns about technical expertise, surgical time, potential complications and costs [21]. Although the robotic system requires learning new technical skills, our study has shown that each subsequent operation was approximately one minute shorter. In fact, the average time for the last 10 robotic cases was $82.9 \mathrm{~min}$ which is quicker than the average time of the conventional group (84.9 min). By the end of these cases, a plateau has not been reached so there is potential for surgical time to decrease further. Redmond et al. [18] similarly reported a statistically significant shorter operating time after the first 35 cases, where operative time was recorded as time of incision to time closure began.

The low numbers of complications in both groups were comparable. It is interesting to note that the conventional group had 3 intraoperative acetabular fractures while the robotic group had none. This could be secondary to the single ream, minimal bone resection technique utilised by the robotic system $[9,22]$ which may decrease the risk of acetabular fractures. Our study also did not find any significant differences in transfusion rates between the groups. It has been suggested that the robotic single-stage reaming of the acetabulum compared to conventional sequential reaming may result in decreased blood loss and hence transfusions [9].

The length of stays in hospital was similar in both group, however once the patients who required inpatient rehabilitation were excluded, the robotic group had a shorter stay in the hospital. There were no changes in rehabilitative protocol that could have allowed patients to be discharged sooner. As only small numbers went to rehabilitation, statistical analysis was not performed on this group, but it is interesting to note that the average length of stay in rehabilitation was also shorter in the robotic group. Larger studies will be required to provide conclusive results.

Although there is a higher initial cost associated with the robot, this could be outweighed by the cost savings down the track. A recent Australian study have reported average inpatient costs following total hip arthroplasty to be approximately $\$ 22,500$, with an average length of stay of 4 days [23]. We are not aware of any current literature describing cost-effectiveness of robot-assisted hip arthroplasty, such as the Markov based analysis on robot-assisted knee arthroplasty [24]. It is also hard to place a monetary value on the better patient reported outcomes reported in the robotic group by Bukowski et al. [9].

Limitations of this study include the retrospective, single surgeon, single institution study design, as well as the small sample size. Future studies with larger sample sizes and prospective study designs could help provide better evidence on robotic hip arthroplasty. Our small sample size did not permit conclusive results on transfusions, complications and LOS in patients that went to rehabilitation. Given the difference in surgical time and length of stays, it may also be useful to conduct a cost-benefit analysis. We are also currently writing a paper that focuses on clinical outcomes which include patient reported outcome measures (PROMs) [25,26].

\section{Conclusion}

Robotic arm assisted THA is a relatively new technology which is gaining popularity. Surgical time is comparable with conventional techniques after the initial learning curve of approximately 35 cases. Compared to conventional THA there is no increased risk of complications or transfusions and interestingly there may be less chance of intraoperative acetabular fractures due to the single ream, minimal bone resection technique. LOS in the robotic group that did not go to rehabilitation was shorter by approximately 1 day. This reduction in LOS, comparable surgical times and potential for less complications may outweigh the increased initial costs associated with the robotic system.

\section{Source of Funding \& Conflicts of Interest}

None

\section{References}

1. Yoo JH, Chang CB, Kang YG, Kim SJ, Seong SC, et al. (2011) Patient expectations of total knee replacement and their association with sociodemographic factors and functional status. J Bone Joint Surg 93: 337-344.

2. Kurtz S, Ong K, Lau E, Mowat F, Halpern M (2007) Projections of primary and revision hip and knee arthroplasty in the United States from 2005 to 2030. J Bone Joint Surg Am 89: 780-785.

3. Bozic K, Kurtz S, Lau E, Ong K, Vail T, et al. (2009) The epidemiology of revision total hip arthroplasty in the United States. J Bone Joint Surg Am 91: 128-133.

4. Kennedy JG, Rogers WB, Soffe KE, Sullivan RJ, Griffen DG, et al. (1998) Effect of acetabular component orientation on recurrent dislocation, pelvic osteolysis, polyethylene wear, and component migration. J Arthroplasty 13: 530-534.

5. Yamaguchi M, Akisue T, Bauer TW, Hashimoto Y (2000) The spatial location of impingement in total hip arthroplasty. J Arthroplasty 15: 305-313.

6. Ali-Khan MA, Brakenbury PH, Reynolds IS (1981) Dislocation following total hip replacement. J Bone Joint Surg 63: 214-218.

7. Leslie IJ, Williams S, Isaac G, Ingham E, Fisher J (2009) High cup angle and microseparation increase the wear of hip surface replacements. Clin Orthop Relat Res 467: 2259-2265.

8. de Steiger RN, Liu YL, Graves SE (2015) Computer navigation for total knee arthroplasty reduces revision rate for patients less than sixty-five years of age. J Bone Joint Surg 97: 635-642.

9. Bukowski B, Anderson P, Khlopas A, Chughtai M, Mont MR, et al. (2016) Improved Functional Outcomes with Robotic Compared with Manual Total Hip Arthroplasty. Surg Technol Int 29: 303-308.

10. Newman J, Carroll K, Cross M (2014) Robotic-assisted total hip arthroplasty: a critical appraisal. Robotic Surgery: Research and reviews 1: 37-42.

11. Tarwala R, Dorr LD (2011) Robotic assisted total hip arthroplasty using the MAKO platform. Curr Rev Musculoskelet Med 4: 151-156.

12. Dorr LD, Deshmane P (2009) Precision surgery. Orthopedics 32: 659-661.

13. Domb B, El Bitar Y, Sadik A, Stake C, Botser I (2014) Comparison of Robotic-assisted and conventional acetabular cup placement in THA: A Matched-pair controlled study. Clin Orthop Relat Res 472: 329-336.

14. Nakamura N, Sugano N, Nishii T, Kakimoto A, Miki H (2010) A comparison between robotic-assisted and manual implantation of cementless total hip arthroplasty. Clin Orthop Relat Res 468: 1072-1081. 
Citation: Heng YY, Gunaratne R, Ironside C, Taheri A (2018) Conventional vs Robotic Arm Assisted Total Hip Arthroplasty (THA) Surgical Time, Transfusion rates, Length of Stay, Complications and Learning Curve. J Arthritis 7: 272. doi:10.4172/2167-7921.1000272

Page 4 of 4

15. Illgen R, Bukowski B, Abiola R, Anderson P, Chughtai M, et al. (2017) Robotic-Assisted Total Hip Arthroplasty: Outcomes at Minimum TwoYear Follow-Up. Surg Technol Int 30: 365-372.

16. Honl M, Dierk O, Gauck C, Carrero V, Lampe F, et al. (2003) Comparison of robotic-assisted and manual implantation of a primary total hip replacement: a prospective study. J Bone Joint Surg 85: 1470-1478.

17. Schulz AP, Seide K, Queitsch C, von Haugwitz A, Meiners J, et al. (2007) Results of total hip replacement using the Robodoc surgical assistant system: clinical outcome and evaluation of complications for 97 procedures. Int J Med Robot 3: 301-306.

18. Redmond J, Gupta A, Hammarstedt J, Petrakos A, Finch N, et al. (2015) The Learning Curve Associated with Robotic-Assisted Total Hip Arthroplasty. The Journal of Arthroplasty 30: 50-54.

19. Lang JE, Mannava S, Floyd AJ, Goddard MS, Smith BP, et al. (2011) Robotic systems in orthopaedic surgery. J Bone Joint Surg Br 93: 1296-1299.

20. Perets I, Walsh J, Close M, Mu B, Yuen L, et al. (2018) Robot-assisted total hip arthroplasty: Clinical outcomes and complication rate. Int J Med Robot 14: e1912.

21. Jacofsky DJ, Allen M (2016) Robotics in Arthroplasty: A Comprehensive Review. J Arthroplasty 31: 2353-2363.
22. Suarez-Ahedo C, Gui C, Martin T, Stake C, Chandrasekaran S, et al. (2017) Robotic-arm assisted total hip arthroplasty results in smaller acetabular cup size in relation to the femoral head size: A matched-pair controlled study. Hip Int 27: 147-152.

23. Peel T, Cheng A, Liew D, Buising K, Lisik J, et al. (2015) Direct Hospital Cost Determinants Following Hip and Knee Arthroplasty. Arthritis Care Res 67: 782-790.

24. Moschetti W, Konopka J, Rubash H, Genuario J (2016) Can RobotAssisted Unicompartmental Knee Arthroplasty be Cost-effective? A Markov Decision Analysis. J Arthroplasty 31: 759-765.

25. Harris WH (1969) Traumatic arthritis of the hip after dislocation and acetabular fractures: treatment by mold arthroplasty. An end-result study using a new method of result evaluation. J Bone Joint Surg Am 51: 737-755.

26. Klassbo M, Larsson E, Mannevik E (2003) Hip disability and osteoarthritis outcome score. An extension of the Western Ontario and McMaster Universities Osteoarthritis Index. Scand J Rheumatol 32: 46-51. 